\title{
A new discrete Hilbert-type inequality involving partial sums
}

\author{
Vandanjav Adiyasuren ${ }^{1}$, Tserendorj Batbold ${ }^{1 *}$ and Laith Emil Azar ${ }^{2}$
}

"Correspondence:

tsbatbold@hotmail.com

${ }^{1}$ Department of Mathematics,

National University of Mongolia,

Ulaanbaatar, Mongolia

Full list of author information is

available at the end of the article

\begin{abstract}
In this paper, we derive a new discrete Hilbert-type inequality involving partial sums. Moreover, we show that the constant on the right-hand side of this inequality is the best possible. As an application, we consider some particular settings.
\end{abstract}

MSC: $26 \mathrm{D} 15$

Keywords: Hilbert-type inequality; Gamma function; The best possible constant; Conjugate exponents

\section{Introduction}

The Hilbert inequality [5] asserts that

$$
\sum_{n=1}^{\infty} \sum_{m=1}^{\infty} \frac{a_{m} b_{n}}{m+n}<\frac{\pi}{\sin (\pi / p)}\left(\sum_{m=1}^{\infty} a_{m}^{p}\right)^{\frac{1}{p}}\left(\sum_{n=1}^{\infty} b_{n}^{q}\right)^{\frac{1}{q}}
$$

holds for non-negative sequences $a_{m}$ and $b_{n}$, provided that $\left(\sum_{m=1}^{\infty} a_{m}^{p}\right)^{\frac{1}{p}}>0$ and $\left(\sum_{n=1}^{\infty} b_{n}^{q}\right)^{\frac{1}{q}}>0$. The parameters $p$ and $q$ appearing in (1) are mutually conjugate, i.e. $\frac{1}{p}+\frac{1}{q}=1$, where $p>1$. In addition, the constant $\frac{\pi}{\sin (\pi / p)}$ is the best possible in the sense that it can not be replaced with a smaller constant so that (1) still holds.

The Hilbert inequality is one of the most interesting inequalities in mathematical analysis. For a detailed review of the starting development of the Hilbert inequality the reader is referred to monograph [5]. The most important recent results regarding Hilbert-type inequalities are collected in monographs [4] and [7].

In 2006, Krnić and Pečarić [6], obtained the following generalization of classical Hilbert inequality.

Theorem 1 Let $p>1, \frac{1}{p}+\frac{1}{q}=1$, and let $2<s \leq 14$. Suppose that $\alpha_{1} \in\left[-\frac{1}{q}, \frac{1}{q}\right), \alpha_{2} \in\left[-\frac{1}{p}, \frac{1}{p}\right)$ and $p \alpha_{2}+q \alpha_{1}=2-s$. If $\sum_{m=1}^{\infty} m^{p q \alpha_{1}-1} a_{m}^{p}<\infty$ and $\sum_{n=1}^{\infty} n^{p q \alpha_{2}-1} b_{n}^{q}<\infty$, then

$$
\sum_{m=1}^{\infty} \sum_{n=1}^{\infty} \frac{a_{m} b_{n}}{(m+n)^{s}}<B\left(1-p \alpha_{2}, p \alpha_{2}+s-1\right)\left(\sum_{m=1}^{\infty} m^{p q \alpha_{1}-1} a_{m}^{p}\right)^{\frac{1}{p}}\left(\sum_{n=1}^{\infty} n^{p q \alpha_{2}-1} b_{n}^{q}\right)^{\frac{1}{q}}
$$

where the constant $B\left(1-p \alpha_{2}, p \alpha_{2}+s-1\right)$ is the best possible.

(c) The Author(s) 2019. This article is distributed under the terms of the Creative Commons Attribution 4.0 International License (http://creativecommons.org/licenses/by/4.0/), which permits unrestricted use, distribution, and reproduction in any medium, provided you give appropriate credit to the original author(s) and the source, provide a link to the Creative Commons license, and indicate if changes were made. 
In the last few years, considerable attention is given to a class of Hilbert-type inequalities where the functions and sequences are replaced by certain integral or discrete operators. For example: in 2013, Azar [3] introduced a new Hilbert-type integral inequality including functions $F(x)=\int_{0}^{x} f(t) d t$ and $g(y)=\int_{0}^{y} g(t) d t$. For some related Hilbert-type inequalities where the functions and sequences are replaced by certain integral or discrete operators, the reader is referred to [1] and [2].

The main objective of this paper is to derive a discrete Hilbert-type inequality involving partial sums, similar to a result of Azar [3]. Such inequality is derived by virtue of inequality (2) and some well-known classical inequalities. As an application, we consider some particular settings.

\section{Preliminaries and lemma}

Recall that the Gamma function $\Gamma(\theta)$ and the Beta function $B(\mu, v)$ are defined, respectively, by

$$
\begin{aligned}
& \Gamma(\theta)=\int_{0}^{\infty} t^{\theta-1} e^{-t} d t, \quad \theta>0 \\
& B(\mu, v)=\int_{0}^{\infty} \frac{t^{\mu-1}}{(t+1)^{\mu+v}} d t, \quad \mu, v>0
\end{aligned}
$$

and they satisfy the following relation

$$
B(\mu, v)=\frac{\Gamma(\mu) \Gamma(v)}{\Gamma(\mu+v)} .
$$

By the definition of the Gamma function, the following equality holds:

$$
\frac{1}{(m+n)^{\lambda}}=\frac{1}{\Gamma(\lambda)} \int_{0}^{\infty} t^{\lambda-1} e^{-(m+n) t} d t
$$

To prove our main results we need the following lemma.

Lemma 2 Let $a_{m}>0, a_{m} \in \ell^{1}, A_{m}=\sum_{k=1}^{m} a_{k}$, then for $t>0$, we have

$$
\sum_{m=1}^{\infty} e^{-t m} a_{m} \leq t \sum_{m=1}^{\infty} e^{-t m} A_{m}
$$

Proof Using Abel's summation by parts formula and the inequality $1-\frac{1}{e^{t}} \leq t$, we have

$$
\begin{aligned}
\sum_{m=1}^{\infty} e^{-t m} a_{m} & =\lim _{m \rightarrow \infty} A_{m} e^{-t(m+1)}+\sum_{m=1}^{\infty} A_{m}\left(e^{-t m}-e^{-t(m+1)}\right) \\
& =\left(1-\frac{1}{e^{t}}\right) \sum_{m=1}^{\infty} e^{-t m} A_{m} \\
& \leq t \sum_{m=1}^{\infty} e^{-t m} A_{m}
\end{aligned}
$$

The lemma is proved. 


\section{Main results}

Theorem 3 Let $p>1, \frac{1}{p}+\frac{1}{q}=1, \lambda>0, a_{m}, b_{n}>0, a_{m}, b_{n} \in \ell^{1}$, define $A_{m}=\sum_{k=1}^{m} a_{k}, B_{n}=$ $\sum_{k=1}^{n} b_{k}$. If $\sum_{m=1}^{\infty} m^{p q \alpha_{1}-1} A_{m}^{p}<\infty$ and $\sum_{n=1}^{\infty} n^{p q \alpha_{2}-1} B_{n}^{q}<\infty$, then

$$
\sum_{m=1}^{\infty} \sum_{n=1}^{\infty} \frac{a_{m} b_{n}}{(m+n)^{\lambda}}<C\left(\sum_{m=1}^{\infty} m^{p q \alpha_{1}-1} A_{m}^{p}\right)^{\frac{1}{p}}\left(\sum_{n=1}^{\infty} n^{p q \alpha_{2}-1} B_{n}^{q}\right)^{\frac{1}{q}}
$$

where $\alpha_{1} \in\left[-\frac{1}{q}, 0\right), \alpha_{2} \in\left[-\frac{1}{p}, 0\right)$ and $p \alpha_{2}+q \alpha_{1}=-\lambda$. In addition, the constant $C=$ $p q \alpha_{1} \alpha_{2} B\left(-p \alpha_{2},-q \alpha_{1}\right)$ is the best possible in (5).

Proof Using (3), the left-hand side of inequality (5) can be expressed in the following form:

$$
\begin{aligned}
\sum_{m=1}^{\infty} \sum_{n=1}^{\infty} \frac{a_{m} b_{n}}{(m+n)^{\lambda}} & =\frac{1}{\Gamma(\lambda)} \sum_{m=1}^{\infty} \sum_{n=1}^{\infty} a_{m} b_{n}\left(\int_{0}^{\infty} t^{\lambda-1} e^{-(m+n) t} d t\right) \\
& =\frac{1}{\Gamma(\lambda)} \int_{0}^{\infty} t^{\lambda-1}\left(\sum_{m=1}^{\infty} e^{-t m} a_{m}\right)\left(\sum_{n=1}^{\infty} e^{-t n} b_{n}\right) d t
\end{aligned}
$$

Now, by applying inequality (4) and equality (3) to the previous equality, we have

$$
\begin{aligned}
\sum_{m=1}^{\infty} \sum_{n=1}^{\infty} \frac{a_{m} b_{n}}{(m+n)^{\lambda}} & \leq \frac{1}{\Gamma(\lambda)} \int_{0}^{\infty} t^{\lambda+1}\left(\sum_{m=1}^{\infty} e^{-t m} A_{m}\right)\left(\sum_{n=1}^{\infty} e^{-t n} B_{n}\right) d t \\
& =\frac{1}{\Gamma(\lambda)} \sum_{m=1}^{\infty} \sum_{n=1}^{\infty} A_{m} B_{n}\left(\int_{0}^{\infty} t^{\lambda+1} e^{-(m+n) t} d t\right) \\
& =\frac{\Gamma(\lambda+2)}{\Gamma(\lambda)} \sum_{m=1}^{\infty} \sum_{n=1}^{\infty} \frac{A_{m} B_{n}}{(m+n)^{\lambda+2}} .
\end{aligned}
$$

Moreover, the last double series represents the left-hand side of the Hilbert-type inequality (2) for $s=2+\lambda$, that is, we have the inequality

$$
\sum_{m=1}^{\infty} \sum_{n=1}^{\infty} \frac{A_{m} B_{n}}{(m+n)^{\lambda+2}}<B\left(1-p \alpha_{2}, p \alpha_{2}+\lambda+1\right)\left(\sum_{m=1}^{\infty} m^{p q \alpha_{1}-1} A_{m}^{p}\right)^{\frac{1}{p}}\left(\sum_{n=1}^{\infty} n^{p q \alpha_{2}-1} B_{n}^{q}\right)^{\frac{1}{q}}
$$

so by (7) we get

$$
\sum_{m=1}^{\infty} \sum_{n=1}^{\infty} \frac{a_{m} b_{n}}{(m+n)^{\lambda}}<C\left(\sum_{m=1}^{\infty} m^{p q \alpha_{1}-1} A_{m}^{p}\right)^{\frac{1}{p}}\left(\sum_{n=1}^{\infty} n^{p q \alpha_{2}-1} B_{n}^{q}\right)^{\frac{1}{q}}
$$

Now we shall prove that the constant factor is the best possible. Assuming that the constant $C$ is not the best possible, then there exists a positive constant $K$ such that $K<C$ and (5) still remains valid if $C$ is replaced by $K$. Further, consider the $\tilde{a}_{m}=m^{-q \alpha_{1}-1-\frac{\varepsilon}{p}}$ and $\tilde{b}_{n}=n^{-p \alpha_{2}-1-\frac{\varepsilon}{q}}$, where $\varepsilon>0$ is sufficiently small number. Then, we have

$$
\tilde{A}_{m}=\sum_{k=1}^{m} \tilde{a}_{k}=\sum_{k=1}^{m} m^{-q \alpha_{1}-1-\frac{\varepsilon}{p}} \leq \int_{0}^{m} x^{-q \alpha_{1}-1-\frac{\varepsilon}{p}} d x=\frac{m^{-q \alpha_{1}-\frac{\varepsilon}{p}}}{-q \alpha_{1}-\frac{\varepsilon}{p}}
$$


and similarly

$$
\tilde{B}_{n}=\sum_{k=1}^{n} \tilde{b}_{k} \leq \frac{n^{-p \alpha_{2}-\frac{\varepsilon}{q}}}{-p \alpha_{2}-\frac{\varepsilon}{q}}
$$

Inserting the above sequences in (5), the right-hand side of (5) becomes

$$
\begin{aligned}
& K\left(\sum_{m=1}^{\infty} m^{p q \alpha_{1}-1} \tilde{A}_{m}^{p}\right)^{\frac{1}{p}}\left(\sum_{n=1}^{\infty} n^{p q \alpha_{2}-1} \tilde{B}_{n}^{q}\right)^{\frac{1}{q}} \\
& \quad \leq K\left(\sum_{m=1}^{\infty} m^{p q \alpha_{1}-1} \frac{m^{-p q \alpha_{1}-\varepsilon}}{\left(-q \alpha_{1}-\frac{\varepsilon}{p}\right)^{p}}\right)^{\frac{1}{p}}\left(\sum_{n=1}^{\infty} n^{p q \alpha_{2}-1} \frac{n^{-p q \alpha_{2}-\varepsilon}}{\left(-p \alpha_{2}-\frac{\varepsilon}{q}\right)^{q}}\right)^{\frac{1}{q}} \\
& =\frac{K}{\left(-q \alpha_{1}-\frac{\varepsilon}{p}\right)\left(-p \alpha_{2}-\frac{\varepsilon}{q}\right)}\left(\sum_{m=1}^{\infty} m^{-1-\varepsilon}\right)^{\frac{1}{p}}\left(\sum_{n=1}^{\infty} n^{-1-\varepsilon}\right)^{\frac{1}{q}} \\
& =\frac{K}{\left(-q \alpha_{1}-\frac{\varepsilon}{p}\right)\left(-p \alpha_{2}-\frac{\varepsilon}{q}\right)}\left(1+\sum_{m=2}^{\infty} m^{-1-\varepsilon}\right) \\
& \leq \frac{K}{\left(-q \alpha_{1}-\frac{\varepsilon}{p}\right)\left(-p \alpha_{2}-\frac{\varepsilon}{q}\right)}\left(1+\int_{1}^{\infty} x^{-1-\varepsilon} d x\right) \\
& =\frac{K(1+\varepsilon)}{\varepsilon\left(-q \alpha_{1}-\frac{\varepsilon}{p}\right)\left(-p \alpha_{2}-\frac{\varepsilon}{q}\right)} .
\end{aligned}
$$

Now, let us estimate the left-hand side of inequality (5). Namely, by inserting the above defined sequences $\tilde{a}_{m}$ and $\tilde{b}_{n}$ in the left-hand side of inequality (5), we get the inequality

$$
\begin{aligned}
\sum_{m=1}^{\infty} \sum_{n=1}^{\infty} \frac{\tilde{a}_{m} \tilde{b}_{n}}{(m+n)^{\lambda}} & =\sum_{m=1}^{\infty} \sum_{n=1}^{\infty} \frac{m^{-q \alpha_{1}-1-\frac{\varepsilon}{p}} n^{-p \alpha_{2}-1-\frac{\varepsilon}{q}}}{(m+n)^{\lambda}} \\
& \geq \int_{1}^{\infty} \int_{1}^{\infty} \frac{x^{-q \alpha_{1}-1-\frac{\varepsilon}{p}} y^{-p \alpha_{2}-1-\frac{\varepsilon}{q}}}{(x+y)^{\lambda}} d x d y \\
& =\int_{1}^{\infty} x^{-1-\varepsilon} \int_{1 / x}^{\infty} \frac{u^{-p \alpha_{2}-1-\frac{\varepsilon}{q}}}{(1+u)^{\lambda}} d u d x \\
& =\int_{1}^{\infty} x^{-1-\varepsilon}\left(\int_{0}^{\infty} \frac{u^{-p \alpha_{2}-1-\frac{\varepsilon}{q}}}{(1+u)^{\lambda}} d u-\int_{0}^{1 / x} \frac{u^{-p \alpha_{2}-1-\frac{\varepsilon}{q}}}{(1+u)^{\lambda}} d u\right) d x \\
& \geq \int_{1}^{\infty} x^{-1-\varepsilon}\left(\int_{0}^{\infty} \frac{u^{-p \alpha_{2}-1-\frac{\varepsilon}{q}}}{(1+u)^{\lambda}} d u-\int_{0}^{1 / x} u^{-p \alpha_{2}-1-\frac{\varepsilon}{q}} d u\right) d x \\
& =\frac{1}{\varepsilon} \cdot B\left(-p \alpha_{2}-\frac{\varepsilon}{q},-q \alpha_{1}+\frac{\varepsilon}{q}\right)-\frac{1}{\left(p \alpha_{2}+\frac{\varepsilon}{q}\right)\left(p \alpha_{2}-\frac{\varepsilon}{p}\right)} .
\end{aligned}
$$

It follows from inequalities (8) and (9) that

$$
B\left(-p \alpha_{2}-\frac{\varepsilon}{q},-q \alpha_{1}+\frac{\varepsilon}{q}\right)-\frac{\varepsilon}{\left(p \alpha_{2}+\frac{\varepsilon}{q}\right)\left(p \alpha_{2}-\frac{\varepsilon}{p}\right)} \leq \frac{K(1+\varepsilon)}{\left(-q \alpha_{1}-\frac{\varepsilon}{p}\right)\left(-p \alpha_{2}-\frac{\varepsilon}{q}\right)} .
$$


Now, letting $\varepsilon \rightarrow 0+$, relation (10) yields a contradiction with the assumption $K<C$. So the constant $C$, in inequality (5) is the best possible.

Considering Theorem 3, equipped with parameters $\lambda=1, \alpha_{1}=-\frac{1}{q^{2}}, \alpha_{2}=-\frac{1}{p^{2}}$, we obtain the following result.

Corollary 4 Let $p>1, \frac{1}{p}+\frac{1}{q}=1$, and let $a_{m}, b_{n}>0$ with $a_{m}, b_{n} \in \ell^{1}$. If $\sum_{m=1}^{\infty} m^{-p} A_{m}^{p}<\infty$ and $\sum_{n=1}^{\infty} n^{-q} B_{n}^{q}<\infty$, then

$$
\sum_{m=1}^{\infty} \sum_{n=1}^{\infty} \frac{a_{m} b_{n}}{m+n}<\frac{\pi}{p q \sin (\pi / p)}\left(\sum_{m=1}^{\infty}\left(\frac{A_{m}}{m}\right)^{p}\right)^{\frac{1}{p}}\left(\sum_{n=1}^{\infty}\left(\frac{B_{n}}{n}\right)^{q}\right)^{\frac{1}{q}}
$$

where the constant $\frac{\pi}{p q \sin (\pi / p)}$ is the best possible.

Remark 5 It should be noticed here that if sequences $a_{m}, b_{n} \in \ell^{1}$ such that $\sum_{m=1}^{\infty} a_{m}^{p}<\infty$ and $\sum_{n=1}^{\infty} b_{n}^{q}<\infty$, inequality (11) provides refinement of the Hilbert inequality. Indeed, by Hardy's inequality, the series $\sum_{m=1}^{\infty}\left(\frac{A_{m}}{m}\right)^{p}$ and $\sum_{n=1}^{\infty}\left(\frac{B_{n}}{n}\right)^{q}$ are converge. So, inequality (11) holds. The Hilbert inequality becomes after applying Hardy's inequality on the right-hand side of inequality (11).

Letting $\alpha_{1}=\alpha_{2}=\frac{-\lambda}{p q}$ in Theorem 3, we can obtain the following Hilbert-type inequality.

Corollary 6 Let $p>1, \frac{1}{p}+\frac{1}{q}=1$, and let $0<\lambda \leq \min \{p, q\}, a_{m}, b_{n}>0$ with $a_{m}, b_{n} \in \ell^{1}$. If $\sum_{m=1}^{\infty} m^{-\lambda-1} A_{m}^{p}<\infty$ and $\sum_{n=1}^{\infty} n^{-\lambda-1} B_{n}^{q}<\infty$, then

$$
\sum_{m=1}^{\infty} \sum_{n=1}^{\infty} \frac{a_{m} b_{n}}{(m+n)^{\lambda}}<\frac{\lambda^{2}}{p q} B\left(\frac{\lambda}{q}, \frac{\lambda}{p}\right)\left(\sum_{m=1}^{\infty} m^{-\lambda-1} A_{m}^{p}\right)^{\frac{1}{p}}\left(\sum_{n=1}^{\infty} n^{-\lambda-1} B_{n}^{q}\right)^{\frac{1}{q}},
$$

where the constant $\frac{\lambda^{2}}{p q} B\left(\frac{\lambda}{q}, \frac{\lambda}{p}\right)$ is the best possible.

\section{Conclusion}

In the present study, we have established a discrete Hilbert-type inequality involving partial sums. Moreover, we have proved that the constant on the right-hand side of this inequality is the best possible. As an application, we considered some particular settings.

\section{Funding}

The authors would like to thank the National University of Mongolia for supporting this research.

\section{Competing interests}

The authors declare that they have no competing interests.

Authors' contributions

All authors read and approved the final version of the manuscript.

\section{Author details}

'Department of Mathematics, National University of Mongolia, Ulaanbaatar, Mongolia. ${ }^{2}$ Department of Mathematics, Al al-Bayt University, Mafraq, Jordan.

\section{Publisher's Note}

Springer Nature remains neutral with regard to jurisdictional claims in published maps and institutional affiliations. 
References

1. Adiyasuren, V., Batbold, Ts., Krnić, M.: On several new Hilbert-type inequalities involving means operators. Acta Math. Sin. Engl. Ser. 29, 1493-1514 (2013)

2. Adiyasuren, V., Batbold, Ts., Krnić, M.: Multiple Hilbert-type inequalities involving some differential operators. Banach J. Math. Anal. 10(2), 320-337 (2016)

3. Azar, L.E.: Two new forms of Hilbert-type integral inequality. Math. Inequal. Appl. 17(3), 937-946 (2014)

4. Batbold, Ts., Krnić, M., Pečarić, J., Vuković, P.: Further Development of Hilbert-Type Inequalities. Element, Zagreb (2017)

5. Hardy, G.H., Littlewood, J.E., Pólya, G.: Inequalities, 2nd edn. Cambridge University Press, Cambridge (1967)

6. Krnić, M., Pečarić, J.: Extension of Hilbert's inequality. J. Math. Anal. Appl. 324(1), 150-160 (2006)

7. Krnić, M., Pečarić, J., Perić, I., Vuković, P.: Recent Advances in Hilbert-Type Inequalities. Element, Zagreb (2012)

\section{Submit your manuscript to a SpringerOpen ${ }^{\circ}$ journal and benefit from:}

- Convenient online submission

- Rigorous peer review

- Open access: articles freely available online

- High visibility within the field

- Retaining the copyright to your article

Submit your next manuscript at $\boldsymbol{\Delta}$ springeropen.com 